\title{
Factors Affecting the Outcome in the Management of Cervicofacial Infection
}

\author{
Mohammed Kamal Abbas Rashwan MSC, Tarek El Sayed ftohy MD, Ahmed Essa \\ Ahmed MD, Kamal A-A.M. Hassanein ELSherkawy FRCS,Ph.D.
}

Corresponding author: Mohamed Kamal Abbas Rashwan, Faculty of medicine, Sohag university

Gmail: narutooo2013@gmail.com,Mobile:01003848370

\begin{abstract}
Introduction Facial cellulitis and abscesses are common in public health problems and early recognition and management is critical, as they are becoming more severe with a noticeable increase in hospitalization. Aim of work detection of factor affecting outcome in the management of cervicofacial infections and complication to reduce associated mortality and morbidity. Patient and method This study was a prospective study which was conducted at Sohag University Hospital, General Surgery Department. It included patients with cervicofacial infection of odontogenic or non-odontogenic origin either localized or diffuse infection attended to at the Oral and Maxillofacial Surgery Clinic or the Accident and Emergency Unit from October 2016 to October 2017. Results This study included 35 cases, the submandibular space was the most common single site affected (11$34.29 \%$ ), followed by bilateral or multi- space involvement presented (13-37.14\%) of study patients, Diabetes mellitus was the most common associated co- morbidity among study population (12-34.29\%), and other systemic diseases (6-17.14\%). Conclusion Diabetes, bad general condition, impaired laboratory parameters including an elevated blood sugar level and metabolic acidosis at time of admission were found to be significant risk factors for development of post intervention complications and/ or delayed recovery.
\end{abstract}

Keywords: cervicofacial infection, deep neck space infection, DM., odontogenic infection, trismus.

\section{Introduction}

Infection is one of the most commonly occurring head and neck pathologies. Spread of infection can be predicted by anatomic boundaries. Morbidity and mortality from head and neck infections declined dramatically over the past 60 years since the advent of antibiotics, but resistant organisms are spreading into the community 7. Infection involving the orbit, lateral pharyngeal space, or multi facial and oral floor phlegmon and deep neck spaces are known as cervicofacial infections (CFI). When diagnosis and/or adequate treatment are delayed, such infections can be lifethreatening ${ }^{1}$. Dental caries is the most common etiology, followed by oropharyngeal infections, Para nasal sinusitis, mastoiditis, foreign body infection, malignant necrotic lymph nodes, infected congenital cysts, cervical lymphadenitis and others ${ }^{\mathbf{1 0}}$. CFI are common in poor patients lacking proper health resources ${ }^{17}$. Many risk factors could influence the outcome of odontogenic and non-odontogenic infections, various local and systemic factors have been reported. These included age, number of fascial spaces involved ${ }^{\mathbf{1 8}}$, socioeconomic status, smoking 4, patients' systemic health especially diabetes mellitus $\mathbf{9 , 1 2}^{\mathbf{9}}$, space proximity to airway ${ }^{\mathbf{1 6}}$, level of education and the nature of medical practice ${ }^{6}$. The 
treatment of odontogenic infections is based on two main elements: surgical treatment and antibiotic therapy. Antibiotics are essential adjunct and are indicated when there are clear signs of systemic involvement such as pyrexia, lymphadenopathy, difficulty in swallowing, and lockjaw ${ }^{5}$.

\section{Patients and Methods:}

\section{Study design:}

This study was a prospective nonrandomized study which was conducted at Sohag University Hospital, General Surgery Department. It included (35) cases with cervicofacial infection of odontogenic or non-odontogenic origin either localized or diffuse infection attended to at the Oral and Maxillofacial Surgery Clinic or the Accident and Emergency Unit from October 2016 to October 2017. All cases are committed a clear informed consent and the study had been approved by the Sohag Medical Ethics Committee.

\section{Study population:}

The patient clinical data were reviewed including: patient data (name, age, sex, occupation, date of complaint, date of admission, address and socioeconomic state), presence of other underlaying comorbidity and the management protocol which was based on empirical intravenous antibiotics treatment with or without surgical intervention (preoperative, operative and post-operative parameters). The complication observed during hospital stay were also documented.

\section{Preoperative parameters:}

The choice of the technique of drainage of the abscess cavity intra oral or extra oral drainage depends on the anatomical site, size of abscess cavity and its extension and clinical evaluation of the patient.

\section{Results}

Emergency laboratory tests as (admission total leucocytic count, admission random blood sugar, admission coagulation profile and serum creatinine and others) emergency radiological investigation especially US., panorama x-ray, CT and MRI scan were performed in some cases.

\section{Post-operative parameters:}

We assessed the early outcomes and late outcomes as:

\section{Early postoperative outcomes include:}

- result of microscopic culture and sensitivity test if done and possible.

- recollection of pus.

- Occurrence of ischemia of edges and presence of necrotic tissue.

- trismus

- oral feeding.

- length of hospital stays.

Late postoperative outcomes (after discharge for one month weekly) include:

- recollection of pus.

- multi sinus developed or not.

Patients were scheduled for follow-up every week for the first month after discharge.

\section{Study outcomes:}

Patient recovery (good or complicated) was considered a primary outcome for the study.

\section{Statistical analysis:}

Data were recorded using Microsoft Excel 2010 program (Microsoft Co, USA). Normality of data distribution was checked by Shapiro Wilk test. The box plots were used to identify and exclude outliers. Mann-Whitney $U$ test was used for comparison of quantitative variables, while Fisher Exact test was used to compare qualitative variables. Data were presented as median $\left(25^{\text {th }}\right.$ and $75^{\text {th }}$ percentiles $)$ or Odds ratio (95\% Confidence interval). $\mathrm{P}$ value $<0.05$ was considered statistically significant. 
Thirty-five patients were recruited during the period of the study. The mean age was 27 (10$65.5)$ years, $18(51.43 \%)$ male and $17(48.57 \%)$ female. $24(68.57 \%)$ patients had low socioeconomic status while the rest had moderate to high status. Urban residents constituted 14 $(40 \%)$ of study population, while $21(60 \%)$ were rural residents. Four $(11.43 \%)$ of our patients were cigarette smokers and $5(14.29 \%)$ of our patients were goza smokers. Figure (1) demonstrates patients' categorization according to site of infection (space affected). Submandibular space figure (3) was the most common single site affected (34.29\%), followed by bilateral or multi- space figure (5) involvement presented (37.14\%) of study patients. Twenty-two (62.86\%) patients were presented with trismus figure (4). As shown in figure (2), Diabetes mellitus was by far the most common associated co- morbidity among study population (34.29\%), while hypertension constituted (8.57\%) and other systemic diseases $6(17.14 \%)$.

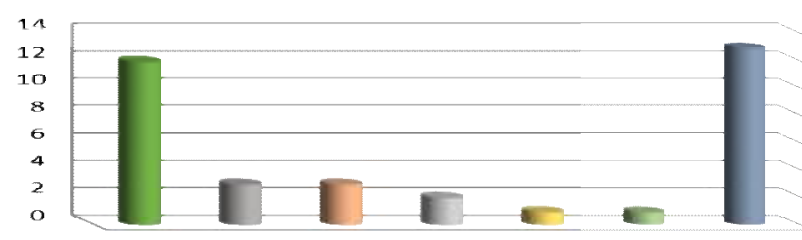

Fig. (1): demonstrates patients" categorization according to site of infection

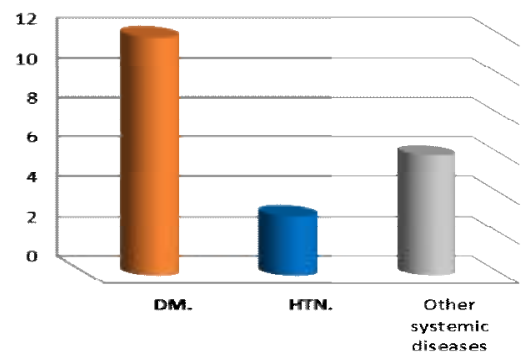

Fig- (2): Prevalence of Associated co-morbidities

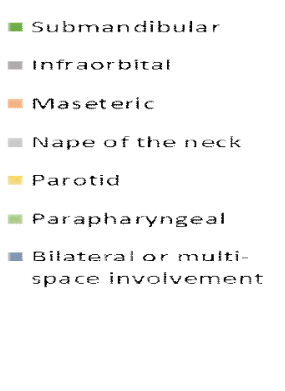

\section{.}
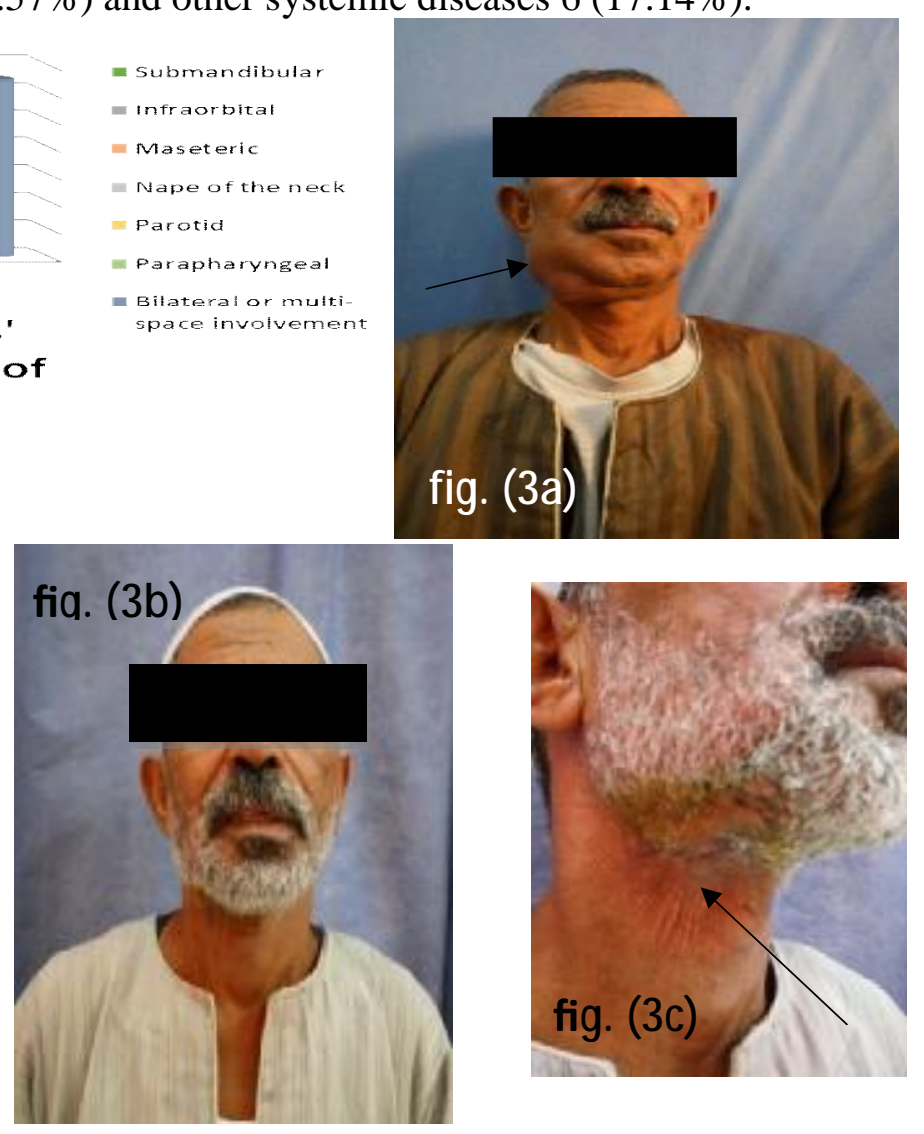

Fig. (3a) Male patient, 65yrs., diabetic, Presented with RT. Submandibular space infection (arrow).

Fig. $(\mathbf{3 d}, \mathbf{c})$ see the same patient post-operative after drainage of the abscess (arrow). (fourth week of follow up).

Fig. (4) Female patient, 40yrs., diabetic, show sever form of trismus (about $0.5 \mathrm{~cm}$.).

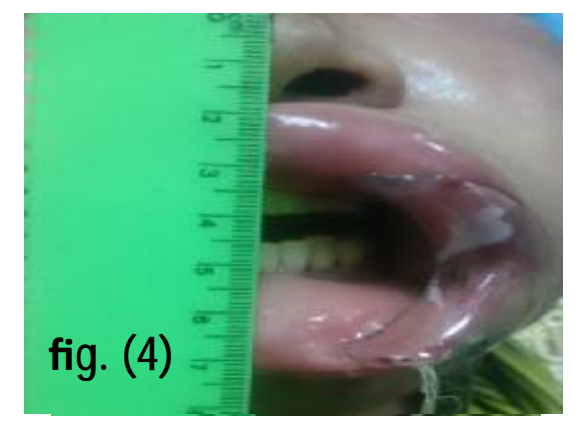



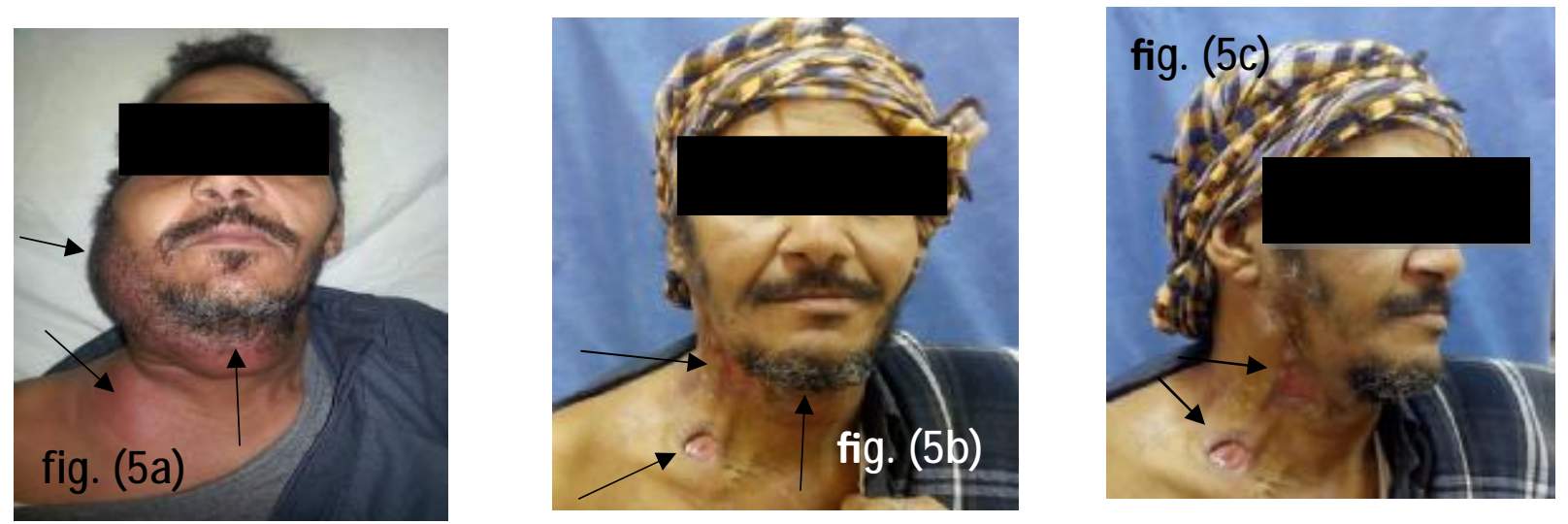

Fig. (5a) Male patient, 50yrs., diabetic, Presented with RT. Submandibular, submental, masseteric and supraclavicular space neck infections (arrows).

Fig. (5d, c) see the same patient post-operative after drainage of the abscess (arrows). (third week of follow up).

So, to investigate risk factors in the outcomes patients in this study, we must get focus in the patients whose got complicated recovery $[n=16,(46 \%)]$. Table (1) shows identified risk factors for complicated recovery. Prevalence of diabetes was significantly higher $[\mathrm{n}=$ 9, (56.25\%)], $(\mathbf{P}=\mathbf{0 . 0 2})$, these patient's median hospital stay was longer by mean of (4.65 $\%)$ than the non-diabetic patients. Pulse was significantly higher [110(110- 120) BPM], $(\mathbf{P}=\mathbf{0 . 0 3})$. Respiratory rate was significantly higher $[25(23-27) \mathrm{CPM}],(\mathbf{P}=\mathbf{0 . 0 0 2})$. Incidence of impaired arterial blood gases was also significantly higher $[n=3$, (18.75\%)], (P=0.04).

\begin{tabular}{|c|c|c|c|c|c|}
\hline \multicolumn{6}{|c|}{ Table (1): Identified risk factors for complicated recovery } \\
\hline Variables & $\begin{array}{l}\text { Good } \\
\text { recovery }(n= \\
19)\end{array}$ & $\begin{array}{l}\text { Complicated } \\
\text { recovery } \\
\quad(n=16)\end{array}$ & $\begin{array}{l}\text { Odds ratio } \\
(95 \% \text { CI })\end{array}$ & $P$ value & Test used \\
\hline DM. & $3(15.79 \%)$ & $9(56.25 \%)$ & $.14(.03-.7)$ & 0.02 & Fisher Exact test \\
\hline Impaired ABG & $0(0 \%)$ & $3(18.75 \%)$ & $\mathbf{0}$ & 0.04 & \\
\hline Pulse (PBM) & $110(100,110)$ & $110(110-120)$ & $\ldots \ldots$ & 0.03 & Mann Whitney u test \\
\hline R.R.(C/M) & $23(23,23)$ & $25(23-27)$ & ...... & 0.002 & \\
\hline RBS (mg/ dl) & $140(130,175)$ & $276(137.5-418.5)$ & ...... & 0.02 & \\
\hline
\end{tabular}

\section{Discussion}

Our study aimed to investigate factors affecting the outcome in the management of cervicofacial infections and patient recovery (either uncomplicated or complicated) following management (either conservative or surgical) was considered a primary outcome for the study.
Regarding patients' demographics and study population characteristics, both genders were found to be nearly equally affected in our study. However, there was a higher prevalence of rural residents and those with low socioeconomic status. These findings are 
consistent with what was previously reported ${ }^{4,6}$. recovery. This finding emphasizes the rule of patient general condition in predicting outcome The submandibular space was found to be theof management of CFI. Also, this should be put most common site of infection among our studyinto consideration when choosing intervention population. Many other studies reported similarmodalities for those patients, either conservative findings ${ }^{2,3,5,6}$. or surgical.

While $50 \%$ of patients with complicatedLaboratory investigations were also important to recovery had a bilateral or multi- spacebe put into consideration in our study. Presence involvement, only $26.32 \%$ of patients recoveredof elevated random blood sugar level and well had a similar condition. Even though, thismetabolic acidosis were both a significant risk finding was not statistically significant.

factors for complicated recovery. Furthermore, Regarding patients' symptomatology, patientsa higher total Leukocytic count and lower Red who had complicated recovery were found toblood cells and platelet counts were observed have a higher incidence of severe symptomsamong patients with complicated recovery but (trismus, odynophagia and dysphagia) at time offailed to reach a statistical significance. This presentation. A recent study has found a positivepositive correlation between CFI patients' correlation between severity of trismus andimpaired laboratory parameters and their duration of hospitalization ${ }^{3}$, which is consistentdelayed recovery was recently reported in with our finding. similar studies ${ }^{\mathbf{3 , 6}}$. Culture and sensitivity has Patient's medical history and general health arebeen a routine practice in our hospital and was another 2 important items to be considered. Inperformed to all patients in the study. Patients our study, Diabetes was found to be awith a positive culture for both aerobes and significant risk factor for complicated recoveryanaerobes were found to be at a slightly higher following management of CFI. These findingsrisk of suffering from recovery complications. are consistent with what was previouslyThis mixed pattern of infection was found to be reported ${ }^{\mathbf{8 , 9 , 1 5}}$. Prevalence of other systemiccommon among CFI patients, especially those diseases was also higher among those patientswith odontogenic infections ${ }^{\mathbf{6}}$.

but did not reach a statistical significance. Regarding intervention data, all our patients An interesting correlation was found in ourincluded in the study had a CFI that necessitated study between patients' vital signs at time ofoperative intervention, except 2 patients who admission and their post intervention recovery.were managed conservatively and had good Patients with tachycardia and tachypnea had arecovery. While a recent significant higher risk of complicated

survey has revealed that only $40 \%$ of UKmost common antibiotic given in overall maxillofacial surgeons would choose operativepatients. This finding is consistent with what intervention if a single fascial space is affectedwas previously reported ${ }^{\mathbf{3 , 1 1}}$. Most of patients by edema and no pus ${ }^{\mathbf{1 1}}$, most of our patientswho received $4^{\text {th }}$ generation cephalosporin + were actually presented with abscess formationmetronidazole had a complicated recovery. This or affection of more than one fascial space.was due to the relatively bad general condition, Neither intraoperative drainage maneuver norpresence of co- morbidities and poly- microbial performance of tooth extraction was found tonature of CFI in these patients and not related to correlate with patient recovery in our study.the antibiotic itself.

Antibiotics were started empirically thenSummary and conclusion modified according to results of culture andManagement of CFI represents a challenge that sensitivity. Amoxicillin- clavulanic acid was thefaces maxillofacial surgeons, with no consistent 
widely implied management protocols andand poly- microbial culture. Although none of many factors affecting the outcome of thisthose factors reached a statistical significance, management.

they were reported by other studies and hence Many assessed parameters in our study wereworth to be mentioned.

founded to be significant risk factors for In conclusion, this study investigated factors complicated recovery following CFIgoverning outcome of CFI management, management. Among them, Diabetes seems toadopting post intervention patients' recovery as be the most important single one. Elevateda primary outcome. Diabetes, bad general random blood sugar and metabolic acidosis arecondition, impaired laboratory parameters another 2 important factors. including an elevated blood sugar level and Patients' bad general condition at time ofmetabolic acidosis at time of admission were admission was also found to negatively correlatefound to be significant risk factors for with post intervention recovery. Patients withdevelopment of post intervention complications uncontrolled diabetes affecting their generaland/ or delayed recovery. According to these health are- therefore- a high risk group that needfinding, were recommend to consider patients to be managed meticulously and properly whenwith uncontrolled diabetes affecting their presented with CFI. general health a high-risk group that need to be Other possible risk factors are: severity ofmanaged meticulously and properly when symptoms at time of presentation, bilateral orpresented with CFI. multi- space involvement, leukocytosis, anemia

\section{References}

1.Adekeye EO and Adekeye JO. The pathogenesis and microbiology of idiopathic cervicofacial abscesses. J Oral Maxillofac Surg. 1982; 40:100-6

health institution: a retrospective analysis of 77 cases. Journal of the Korean Association of Oral and Maxillofacial Surgeons. 2015; 41 293-8

2.Al-Malik $M$ and Al-Sarheed M. Pattern of7.Herr RD, Serious Soft Tissue Infections of the management of oro-facial infection in children: A retrospective. Saudi Journal of Biological Sciences. 2017; 24:1375-9 Head and Neck, American Family Physician, September 1991;60:830-34

3.Aloosi S, Abdullah $\mathrm{H}$ and Yaqub F . Factors influencingManagement Outcome of Hospitalised Patients with Odontogenic Infection.2017; vol 4

4.Bakathir A A, Moos K F, Ayoub A F . et al. Factors Contributing to the Spread of Odontogenic Infections: A prospective pilot study. Sultan Qaboos University medical9.Kamat R D, Dhupar V, Akkara F.et al. A journal. 2009; 9 296-304

8.Juncar M, Popa A R, Baciut M F. et al. Evolution assessment of head and neck infections in diabetic patients--a case control study. Journal of cranio-maxillo-facial surgery : official publication of the European Association for Cranio-Maxillo-Facial Surgery. 2014; 42 498-502 comparative analysis of odontogenic maxillofacial infections in diabetic and

5.Bakir S, Tanriverdi M H, Gun R. et al. Deep neck space infections: a retrospective review of 173 cases. American journal of otolaryngology. nondiabetic patients: an institutional study. J Korean Assoc Oral Maxillofac Surg. 2015; 41 $176-80$ 2012; 33 56-63

10.Karkos PD, Leong SC, Beer $\mathrm{H}$, et al.

6.Fomete B, Agbara R, Osunde D O. et al. Challenging airways in deep neck space Cervicofacial infection in a Nigerian tertiary infections. Am J Otolaryngol 2007; 28:415e8. 
11.McDonald C, Hennedige A, Henry A.et al. Management of cervicofacial infections: a12.Peters E S, Fong B, Wormuth D W . et al. Risk survey of current practice in maxillofacial units in the UK. British Journal of Oral and Maxillofacial Surgery. 2017; 55 940-5

ial infections. Journal of oral and maxillofacial surgery : official journal of the American Association of Oral and Maxillofacial Surgeons. 1996; 54 1386-91; discussion 91-2.

13.Bakathir AA, Moos KF, Ayoub AF, et al . Factors Contributing to the Spread of Odontogenic Infections: A prospective pilot study. Sultan Qaboos Univ Med J 2009;9(3):296-304. factors affecting hospital length of stay in patients with odontogenic maxillofac phylotypes in advanced noma lesions. J Clin Microbiol. 2002; 40:2187-2191.

16.Sette-Dias AC, Maldonado AJT, Aguiar EGd. et al. Profile of patients hospitalized with odontogenic infections in a public hospital in Belo Horizonte, Brazil. J Clin Exp Dent.2012; 4(5):271-4.

17.Wang J, Ahani A and Pogrel MA. A five-year retrospective study of odontogenic

14.Cachovan G, Nergiz I, Thuss U. et al. maxillofacial infections in a large urban public Penetration of moxifloxacin into rat hospital. Int J Oral Maxillofacial Surg. 2005; mandibular bone and soft tissue. Acta Odontol 34:646-9

Scand (2009); 67(3):182-6.

18.Zamiri B, Bron. HS, Hill. HS. et al. Prevalence of Odontogenic Deep Head and Neck Spaces

15.Paster BJ, Falkler JW Jr, Enwonwu CO, et al. Infection and its Correlation with Length of Prevalent bacterial species and novel Hospital Stay. Shiraz Univ Dent J 2012; 13(1):29-35. 